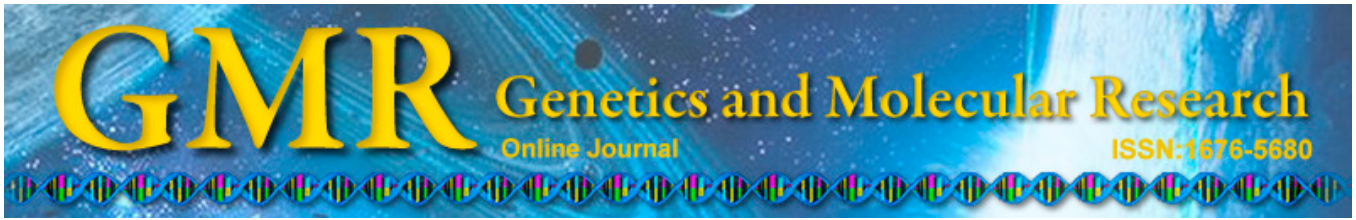

\title{
Expression and antibody generation of the cancer-testis antigen, BIOT2-S
}

\author{
J.-Y. Wang ${ }^{1 *}$, M. Cao ${ }^{2 *}$, M.-R. Guo ${ }^{1}$, S. Li ${ }^{1}$, X.-F. Yang ${ }^{1}$, M. Wang. ${ }^{1}$, \\ J. Fang ${ }^{2}$ and J. Zhao ${ }^{1}$
}

${ }^{1}$ Key Laboratory of Biological Resources and Ecological Environments of The Chinese Education Ministry, College of Life Science, Sichuan University, Chengdu, China

${ }^{2}$ Key Laboratory of the Sichuan Academy of Medical Sciences, Sichuan Provincial People's Hospital, Chengdu, China

*These authors contributed equally to this study.

Corresponding author: J. Zhao

E-mail: zj804@163.com

Genet. Mol. Res. 14 (3): 8685-8693 (2015)

Received January 27, 2015

Accepted March 26, 2015

Published July 31, 2015

DOI http://dx.doi.org/10.4238/2015.July.31.17

ABSTRACT. Biot2-S is a mouse cancer-testis antigen gene that was
identified using the cross-reactive serological analysis of recombinant
CDNA expression libraries (SEREX) technique in the State Key
Laboratory of Biotherapy, West China Hospital, Sichuan University. To
express BIOT2-S and generate its antibody for further investigation, the
Biot2-S prokaryotic recombinant expression vector Biot2-S/pGEX6P-1
was constructed with Escherichia coli $\mathrm{DH}$ - $\alpha$ as a cloning vector, and
BIOT2-S was expressed in E. coli Rosetta (DE3). The recombinant
BIOT2-S was expressed in the form of an inclusion body and the targeted
recombinant BIOT2-S was produced at the level of approximately $25 \%$
total bacterial proteins after being induced with optimum conditions
$\left(0.2\right.$ mM isopropyl- $\beta$-D-thiogalactopyranoside for 6 h at $37^{\circ} \mathrm{C}$ ). The
target protein was purified by glutathione S-transferase (GST)-trap FF
affinity chromatography and detected by western blot. The purified
recombinant protein was further confirmed by electrospray ionization 
quadrupole time-of-flight mass spectrometry after removal of the GSTtags. Then the purified BIOT2-S was used to immunize adult rabbits to generate its antibody. The antibody was purified and its specificity determined. The titer of the antibody was shown to reach $10^{4}$ and the antibody was demonstrated to be able recognize the corresponding protein in the testes of mouse and chicken; the tumor cell lines CT-26 and S180 also reacted with the antibody. This study provides a valuable foundation for further research on the cancer-testis antigen BIOT2-S.

Key words: Biot2-S; Cancer-testis antigen gene; Polyclonal antibody; Recombinant expression

\section{INTRODUCTION}

Mouse Biot2 is a homologous, highly expressed testis gene screened from a rat testicular cDNA library using cross-reactive SEREX as part of the early research of the State Key Laboratory of Biotherapy, West China Hospital, Sichuan University. In brief, when crossreactive SEREX was used to screen a rat testicular cDNA library with rabbit anti-breast and anti-ovarian cancer sera, a repetitive positive clone was isolated. This cDNA was identified as an unknown mRNA sequence without any information about its biological function; a splice variant was also identified. Both splice forms represented new gene sequences and were submitted to GenBank (AY845220 and AY845219) (Yang et al., 2009).

It has been reported that the Biot2- $L$ gene is highly expressed in the adult mouse testis and that regular changes in its expression occur during testis development, such that the expression level is consistent with the degree of sexual maturation (Wang et al., 2009). This suggests that the Biot2 gene might be involved in testicular development and the formation of spermatogonia. Studies have demonstrated that the formation and development of tumor cells share many common characteristics with those processes in germ cells, including invasiveness, immortality, metastatic capacity, vascular formation, hypomethylation, and the downregulation of major histocompatibility complex genes. The genes that regulate the reproductive process might also in turn participate in the occurrence and development of tumors. The finding that Biot 2 is not expressed in non-testicular normal tissues but is expressed in tumor groups and testicular tissues suggests that it might be a tumor-associated protein (Yang et al., 2009). Comparatively, cancertestis antigens (CTAs) have become a highly researched topic in the study of tumor immunotherapy in recent years since they are only expressed in normal testicular tissues and in a variety of tumor tissues, and can cause immune responses in patients with tumors (Caballero and Chen, 2009; Liang et al., 2011). The BIOT2-S protein might therefore not only play an important role in the differentiation of testis and sperm but also might be one of the potential molecular targets for homologous immunotherapy as a new CTA (Wang et al., 2007).

In order to further investigate the functions of BIOT2-S in vitro and in vivo, including the potential relationship between BIOT2-S expression and tumorigenesis, the development of a BIOT2-S antibody is urgently needed. To generate this, we expressed BIOT2-S in a prokaryotic expression vector and optimized its expression conditions, and then used the purified protein as antigen to immunize rabbits to obtain a BIOT2-S antiserum. We then determined the effectiveness and titer of the antibody. This research lays a foundation for the further study of generative cell and tumor immunotherapy. 


\section{MATERIAL AND METHODS}

\section{Material}

Isopropyl- $\beta$-D-thiogalactopyranoside (IPTG) and Freund's adjuvant were purchased from Sigma Aldrich (St. Louis, MO, USA). The plasmid extraction kit and DNA marker were purchased from Tiangen Biotech (Beijing, China). BamHI, XhoI, T4 DNA ligase, ExTaq DNA polymerase, and all the reagents for polymerase chain reaction (PCR) amplification were purchased from TaKaRa (Otsu, Shiga, Japan). The $p G E M-T$ were purchased from Promega (Madison, WI, USA). Horseradish peroxidase (HRP)-conjugated goat-anti-rabbit secondary antibody was purchased from Beyotime Biotech (Shanghai, China). New Zealand white rabbits were purchased from the Laboratory Animal Center of Sichuan University. All animal care and handling procedures were approved by the Institutional Animal Care and Use Committee of Sichuan University.

\section{Methods}

\section{Construction and identification of Biot2-S/pGEX6P-1}

The Biot2-S gene had previously been attached to the $p G E M-T$ in the early study of our laboratory. The Biot2-S gene was released using restriction enzyme digestion and ligated into the $p G E X 6 P-1$ plasmid using $5^{\prime}-$ Bam HI and $3^{\prime}-X h o I$ restriction sites, and the recombinant plasmid was transformed into Escherichia coli DH5a for propagation. To identify correctly generated recombinant plasmids, single E. coli DH5 $\alpha$ clones were cultured and the purified plasmids digested by one or both of the enzymes and analyzed by agarose gel electrophoresis. Clones were further confirmed by PCR and then the positive recombinant plasmids were sequenced by Invitrogen (Carlsbad, CA, USA).

\section{Expression optimization and solubility analysis of the recombinant protein}

To express the target protein, the recombinant plasmid, Biot2-S/pGEX6P-1 was transformed into bacteria and cultured at $37^{\circ} \mathrm{C}$ in Luria broth (LB) medium containing $50 \mu \mathrm{g} / \mathrm{mL}$ kanamycin. When the absorbance of the bacterial culture was 0.6 at $600 \mathrm{~nm}$, IPTG was added to induce the expression of the recombinant protein. To optimize vector expression, E. coli strains BL21 (DE3) and Rosetta (DE3) were compared. To optimize the IPTG dose for induction, the bacteria were induced with different concentrations of IPTG $(0.1,0.2,0.4,0.8$, and $1.0 \mathrm{mM}$ ) for $6 \mathrm{~h}$. To optimize the temperature of induction, the bacteria were induced with 0.2 $\mathrm{mM}$ IPTG at 20,30 , and $37^{\circ} \mathrm{C}$ for $6 \mathrm{~h}$. These samples were analyzed by $12 \%$ sodium dodecyl sulfate polyacrylamide gel electrophoresis (SDS-PAGE) (Laemmli, 1970).

In order to identify the solubility of the target expressed protein, $100 \mathrm{~mL}$ cells from an induced culture were harvested and washed twice with sterile phosphate buffered saline (PBS), pH 7.4. The cells were resuspended in $10 \mathrm{~mL} \mathrm{PBS,} \mathrm{pH} \mathrm{7.4,} \mathrm{and} \mathrm{sonicated} \mathrm{(on} \mathrm{for} 5$ $\mathrm{s}$, off for $5 \mathrm{~s} ; 199$ cycles) on ice, and then centrifuged for $30 \mathrm{~min}$ at $20,000 \mathrm{rpm}$ at $4^{\circ} \mathrm{C}$. The supernatant and pellet were analyzed by SDS-PAGE. 


\section{Purification and identification of recombinant proteins}

The recombinant protein was identified by western blot using HRP labeled anti-GST; protein samples from a null vector transfection and an E. coli Rosetta (DE3) strain that had not been induced with IPTG were used as controls.

After supersonic lysis (on for $5 \mathrm{~s}$, off for $5 \mathrm{~s} ; 199$ cycles) of $100 \mathrm{~mL}$ bacterial culture on ice, the precipitate containing inclusions was washed three times with buffer ( $2 \mathrm{M}$ urea, 20 $\mathrm{mM}$ Tris- $\mathrm{HCl} \mathrm{pH} 8.0,500 \mathrm{mM} \mathrm{NaCl}, 2 \%$ Triton X-100). Inclusions were suspended with 20 mL Buffer A ( $5 \mathrm{mM}$ imidazole, $1 \mathrm{mM} \beta$-mercaptoethanol, $8 \mathrm{M}$ urea, $20 \mathrm{mM}$ Tris- $\mathrm{HCl} \mathrm{pH}$ 8.0, $500 \mathrm{mM} \mathrm{NaCl}$ ), stirred at $4^{\circ} \mathrm{C}$ overnight, and then centrifuged at $4^{\circ} \mathrm{C}, 15,000 \mathrm{rpm}$ for $10 \mathrm{~min}$ to obtain the supernatant. The supernatants containing the denatured proteins were combined on a glutathione sepharose column and the hybrid bacterial proteins were washed by Buffer A, then the GST label was cleaved by thrombin, and the target protein was collected and tested by SDS-PAGE. In parallel, the purified protein was identified by electrospray ionization (ESI) time-of-flight (TOF) mass spectrometry (MS) and its sequence compared with the BIOT2-S sequence in the protein database MASCOT (Gou et al., 2008).

\section{Generation of antiserum}

Purified BIOT2-S was used to immune New Zealand white rabbits. In the first immune experiment, $0.5 \mathrm{~mL}(800 \mu \mathrm{g})$ BIOT2-S was blended with the same volume of Freund's complete adjuvant and injected into the back of a rabbit by subcutaneous injection. After two weeks, an additional $0.25 \mathrm{~mL}(400 \mu \mathrm{g})$ BIOT2-S was blended with the same volume of Freund's incomplete adjuvant to immunize the rabbits. Immunization was enhanced once a week for 4 weeks.

After one week of immunization, ear marginal vein blood was obtained. The titer of the antiserum was estimated using two-way agar immunodiffusion; when an immune precipitation line could be seen with the naked eye even when the antiserum dilution degree reached $1: 16$, heart blood was retrieved to obtain polyclonal antiserum.

\section{Preparation, purification, and identification of rabbit anti-BIOT2-S antiserum}

The polyclonal antiserum was purified by protein A affinity chromatography. The titer of the purified polyclonal antibody was determined by western blot; for this, the polyclonal antibody was diluted 1:500 to 1:10,000 as the primary antibody utilized to detect 15 or 30 ng purified recombinant protein. The antibody was also reacted against bacterial protein to determine its specificity. To further determine the effectiveness of the antibody, it was used in immunohistochemical analysis (IHC) of mouse testis, and utilized in western blots against testis tissue protein of mouse and chicken, as well as against protein from the tumor cells S180 and CT26.

\section{RESULTS}

\section{Construction and identification of the recombinant plasmid Biot $2-S / p G E X 6 P-1$}

The recombinant plasmid was confirmed by NdeI/BamHI restriction enzyme digestion and the release of a $483 \mathrm{bp}$ target fragment, which was consistent with the results of PCR amplification and sequencing analysis (Figure 1). 


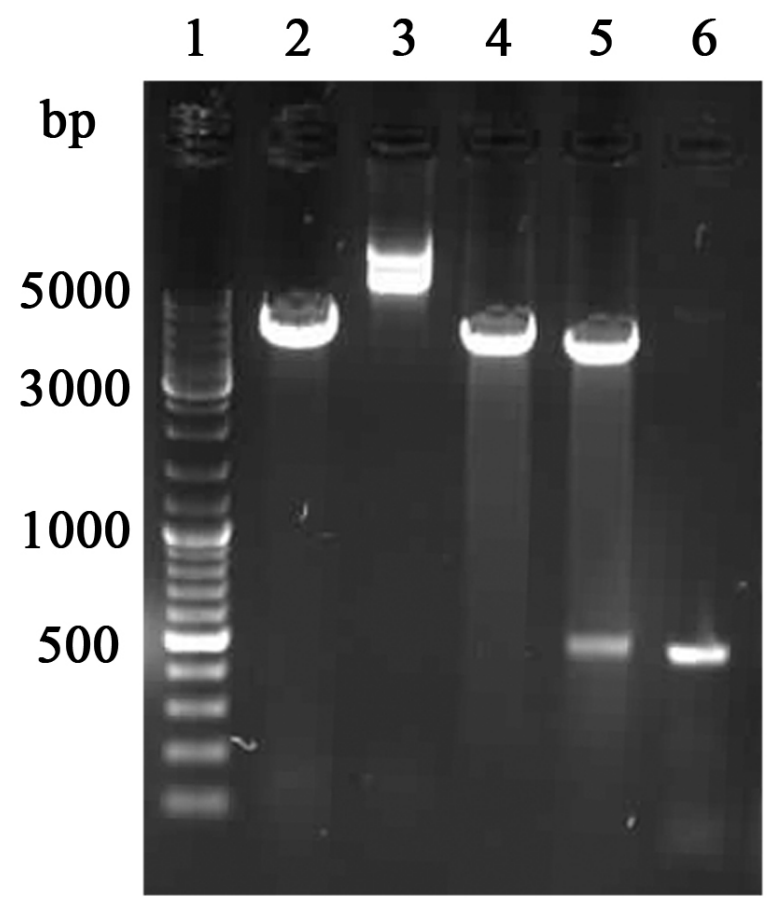

Figure 1. Identification of the recombinant plasmid Biot2-S/pGEX6P-1. Lane 1: $\lambda$ DNA/HindIII digest DNA marker; lane 2: $p G E X 6 P-1$ plasmid; lane 3: Biot2-S/pGEX6P-1 digested with NdeI; lane 4: undigested Biot2-S/ pGEX6P-1; lane 5: Biot2-S/pGEX6P-1 digested with NdeI and BamHI; and lane 6: Bio2-S gene polymerase chain reaction product generated using Biot2-S/pGEX6P-1 as a template.

\section{Optimization of recombinant protein expression and evaluation of its solubility}

The predicted value of approximately $43 \mathrm{kDa}$ for the recombinant protein was confirmed and visualized by SDS-PAGE (Figure 2A); in the figure, the arrow points to the position of the target protein. The results indicated that the GST-tagged BIOT2-S fusion protein was preferentially expressed in E. coli Rosetta (DE3) vs E. coli BL21 (DE3), and that the other optimal conditions for BIOT2-S protein expression were growth at $37^{\circ} \mathrm{C}$ and induction with $0.2 \mathrm{mM}$ IPTG. In Figure 2A, lane 6 shows the recombinant protein induced under the most optimal conditions. After optimized induction, the GST-tagged BIOT2-S fusion protein was produced at the level of approximately $25 \%$ total bacterial proteins.

The GST-tagged BIOT2-S fusion protein was primarily expressed in the total bacterial protein precipitate, implying that the target protein was expressed as inclusion bodies in $E$. coli Rosetta (DE3) (Figure 2B).

\section{Purification and identification of recombinant proteins}

The recombinant proteins were purified by affinity chromatography, the protein GSTtags were removed by thrombin, and the purified product was detected by SDS-PAGE (Figure $3 \mathrm{~A}$ ) and ESI-Q-TOF-MS (Figure 3B). 


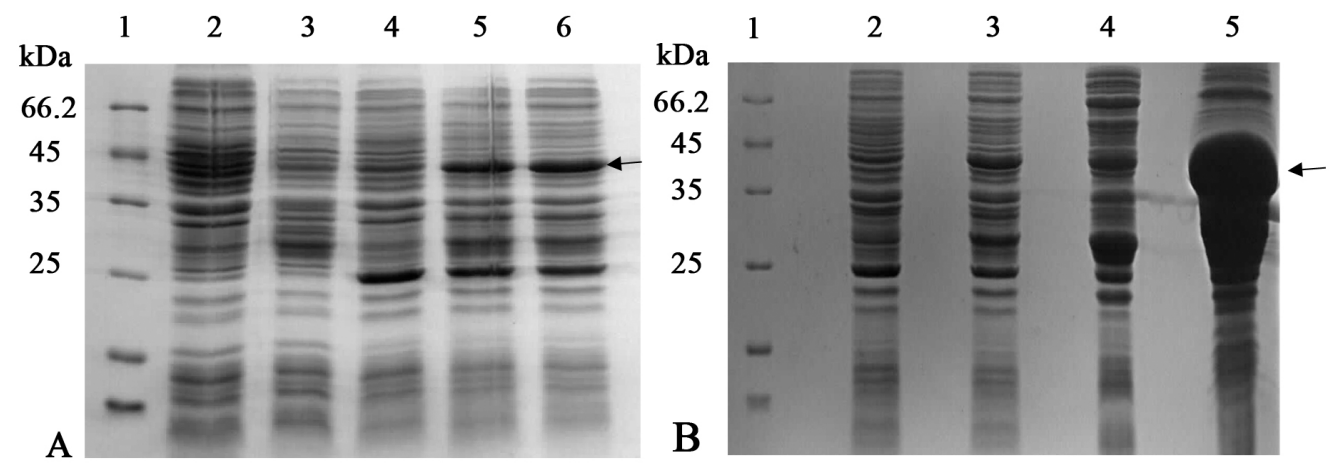

Figure 2. Induction and solubility analysis of the recombinant protein. A. Optimization of recombinant protein induction. Lane 1: protein molecular weight marker; lanes 2 and 3: total bacterial protein of recombinant Escherichia coli BL21 (DE3) before and after IPTG induction; lanes 4 and 5: total bacterial protein of recombinant E. coli Rosetta (DE3) before and after IPTG induction; and lane 6: total bacterial protein of recombinant E. coli Rosetta (DE3) after $0.2 \mathrm{mM}$ IPTG at $37^{\circ} \mathrm{C}$ for $6 \mathrm{~h}$ (optimal conditions). B. Solubility analysis of the recombinant protein. Lane 1: protein molecular weight marker; lanes 2 and 3: total bacterial protein of recombinant E. coli Rosetta (DE3) before and after IPTG induction; lane 4: total bacterial protein supernatant after IPTG induction; and lane 5: total bacterial protein precipitate containing recombinant protein after IPTG induction. IPTG, isopropyl$\beta$-D-thiogalactopyranoside.

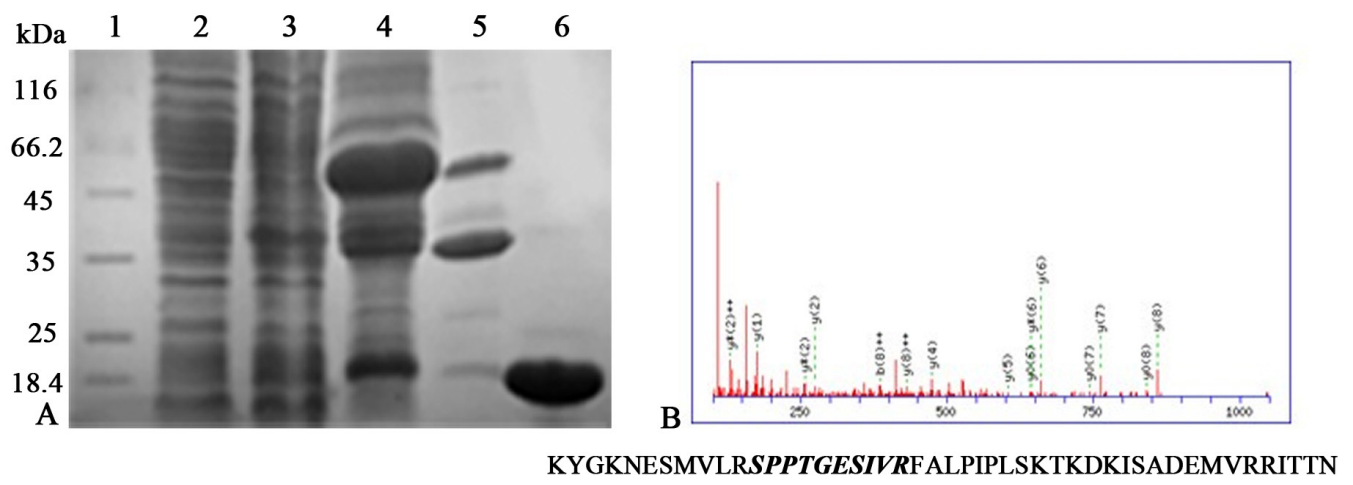

Figure 3. Purification and detection of the recombinant protein. A. Purification of recombinant proteins detected by SDS-PAGE. Lane 1: protein molecular weight marker; lane 2: total bacterial protein of Escherichia coli Rosetta (DE3) $(+)$ without IPTG induction; lane 3: total bacterial protein supernatant of E. coli Rosetta (DE3) (+) after IPTG induction; lane 4: total bacterial protein precipitate of $E$. coli Rosetta (DE3) (+) after IPTG induction; lane 5: recombinant protein after purification; and lane 6: purified target protein after GST-tag removal. B. ESI-Q-TOFMS of BIOT2-S. One of the matched fragments of BIOT2-S. The matched portions within the peptide sequence are indicated with bold italics. SDS-PAGE, sodium dodecyl sulphate-polyacrylamide gel electrophoresis; IPTG, isopropyl- $\beta$-D-thiogalactopyranoside; ESI-Q-TOF-MS, electrospray ionization quadrupole time-of-flight mass spectrometry; GST, glutathione S-transferase.

In Figure 3A, the total amount of hybrid protein in the sample can be seen to have decreased substantially after purification, and the protein was determined to be approximately $17 \mathrm{kDa}$ as was predicted after removal of the GST-tag. Comparison of the protein polypeptide fingerprints obtained by ESI-Q-TOF-MS for the recombinant BIOT2-S with those of the predicted protein sequences identified 10 peptides that obviously matched, with a similarity 
of $99.95 \%$. One of the BIOT2-S matched fragments is displayed in Figure 3B; the matched portions are indicated in bold. Mass spectrometry analysis further confirmed that the purified recombinant protein was the BIOT2-S fusion protein.

\section{Characterization of rabbit anti-BIOT2-S antiserum}

The titer of the antibody obtained from the immunization of rabbits with purified BIOT2-S protein was determined by western blot. As illustrated in Figure 4A, the antibody could detect $15 \mathrm{ng}$ antigen at a dilution of 1:10,000, showing a clear band, indicating that the titer of the antibody was $10^{4}$. Figure $4 \mathrm{~B}$ demonstrates that the antibody detects the induced protein but not bacterial proteins, which confirms the specificity of the antibody. IHC of mouse testis (Figure 5A) revealed that BIOT2-S was located in the cytoplasm. Further western blot analysis determined that the antibody could detect a signal from samples of total protein from mouse and chicken testes (Figure 5B), demonstrating that BIOT2-S is expressed in both mammals and birds; in addition, the antibody could also detect a signal from total protein of the tumor cell lines S180 and CT26 (Figure 5C).

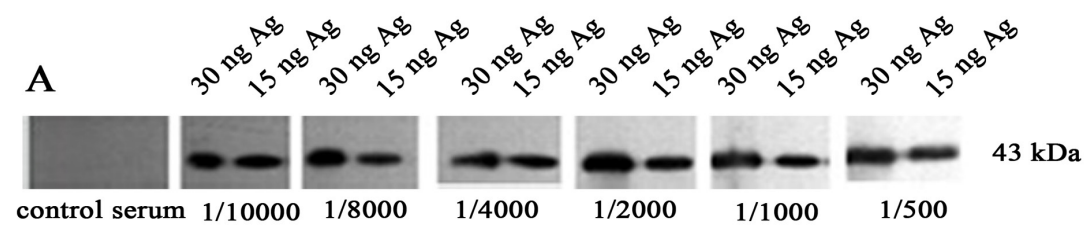

\section{B}

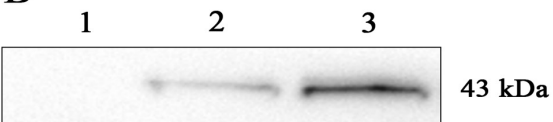

Figure 4. Detection of antibody titer and specificity. A. Western blot results of the purified polyclonal IgG. B. Detection of antibody titer and specificity. Lane 1: total bacterial protein of Escherichia coli Rosetta (DE3) without recombinant plasmid; lane 2: total bacterial protein of E. coli Rosetta (DE3) (+) without IPTG induction; and lane 3: total bacterial protein of E. coli Rosetta (DE3) (+) with IPTG induction. IPTG, isopropyl- $\beta$-Dthiogalactopyranoside.

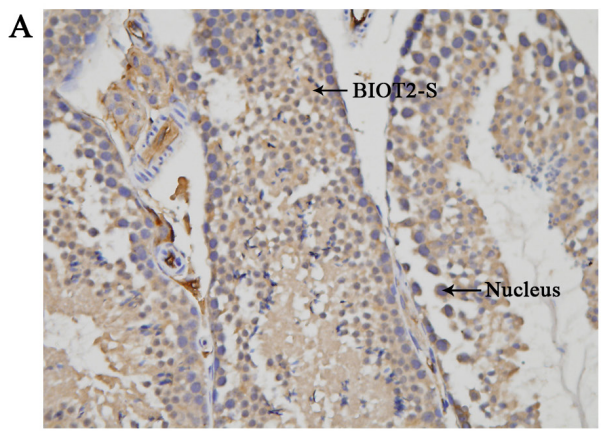

B

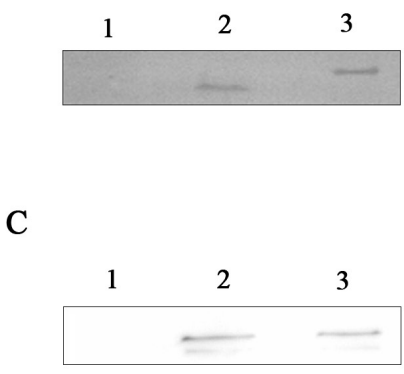

Figure 5. Determination of antibody effectiveness. A. Immunohistochemical analysis of mouse testis. B. Western blot of mouse and chicken testes. Lane 1: control; lane 2: total protein of chicken testicular tissue; and lane 3 total protein of mouse testicular tissue. C. Western blot of tumor cell lines. Lane 1: control; lane 2: total protein of the tumor cell line S180; and lane 3: total protein of the tumor cell line CT26. 


\section{DISCUSSION}

Surgery, radiotherapy, and chemotherapy are the most important methods currently used for cancer treatment in the clinic, but traditional therapies kill healthy cells in addition to cancerous ones, causing side effects such as nausea and vomiting, shin sores and rashes, hair loss, and fatigue (Li et al., 2011a; Fallon, 2013). Such side effects reduce patient quality of life and are harmful to recovery. To solve these problems, researchers have continuously put forward new ideas such as using new materials to replace traditional medicines to improve the effects of treatment (Shen et al., 2014); however, these methods have not fundamentally altered the current state of treatment. Concurrently, the development of tumor immunology methodologies has brought new opportunities for cancer therapy (Goel and Sun, 2014). Tumor associated antigens (TAAs) play an important role in immunology but some TAAs are expressed only in limited types of tumors or only at certain times. It is therefore important to identify new TAAs, such as the telomerase catalytic subunit, that are widely expressed in different kinds of tumor tissue (Vonderheide et al., 1999). In adoptive immunotherapy, the T cell receptor, TCR, plays a crucial role in the recognition of tumor antigens. Tumor specific TCRs can be used to transform normal T cells, turning them into specific cancer killer cells (Johnson et al., 2009). During this process, the choice of antigens such as CTA, which are expressed only in tumor cells, can avoid systemic risk (Scanlan et al., 2002; Li et al., 2011b; Robbins et al., 2011). In addition, CTA can also be used to generate monoclonal antibodies and tumor vaccines. At present, some CTA tumor vaccines including Mage-A1 and A3 have begun to be used in clinical practice, and have achieved a prominent curative effect (Ueda et al., 2007; Lin et al., 2010; Xu et al., 2012). In the process of developing biological immunotherapies, it is also important to find new antigens to resist immune tolerance (He et al., 2003; Lu et al., 2003); thus, the identification of tumor specific antigens is paramount for the development of effective immunotherapeutic strategies for treating human cancers.

As the importance of the protein BIOT2-S as a potential molecular target for future immune therapy has been implicated, and the positive effects of the application of antibodies in disease diagnosis and treatment have been demonstrated (Lasickienè et al., 2011), this research focused on effective BIOT2-S expression and antibody preparation. According to our results, the GST labeled recombinant protein BIOT2-S was primarily expressed in the form of inclusion bodies in E. coli Rosetta (DE3). Purified protein was identified by western blot. Recombinant protein was used to immunize a New Zealand white rabbit to generate an antibody with a titer of $10^{4}$. IHC revealed that the cellular localization of BIOT2-S was cytoplasmic and further western blot analysis indicated that the antibody could detect a signal from testis and tumor cells, and that the association was specific to the BIOT2-S protein. Therefore, this study has not only lain the foundation for BIOT2-S research in tumor immunotherapy, but also for the development of reproductive research as well.

\section{Conflicts of interest}

The authors declare no conflict of interest.

\section{ACKNOWLEDGMENTS}

Research financially supported by the National Natural Science Foundation of China 
(\#31270175, \#81301872). This was also supported by the Sichuan Provincial Research Program for Distinguished Young Scientists, the Program for New Century Excellent Talents in University and the Fundamental Research Funds for the Central Universities (\#2011JQ0018, \#NCET-13-0397, \#2013SCU04B14).

\section{REFERENCES}

Caballero OL and Chen YT (2009). Cancer/testis (CT) antigens: potential targets for immunotherapy. Cancer Sci. 100: 2014-2021.

Fallon MT (2013). Neuropathic pain in cancer. Br. J. Anaesthes. 111: 105-111.

Goel G and Sun WJ (2014). Cancer immunotherapy in clinical practice - the past, present, and future. Chin. J. Cancer. 33: 445-457.

Gou LT, Tong AP, Chen LJ, Tang MH, et al. (2008). Comparative plasma membrane-associated proteomics of immortalized human hepatocytes. Biochemistry (Mosc) 73: 1200-1206.

He QM, Wei YQ, Tian L, Zhao X, et al. (2003). Inhibition of tumor growth with a vaccine based on xenogeneic homologous fibroblast growth factor receptor-1 in mice. J. Biol. Chem. 278: 21831-21836.

Johnson LA, Morgan RA, Dudley ME, Cassard L, et al. (2009). Gene therapy with human and mouse T-cell receptors mediates cancer regression and targets normal tissues expressing cognate antigen. Blood 114: 535-546.

Laemmli UK (1970). Cleavage of structural proteins during the assembly of the head of bacteriophage T4. Nature 227: 680-685.

Lasickienè R, Gedvilaite A, Norkiene M, Simanaviciene V, et al. (2012). The use of recombinant pseudotype virus-like particles harbouring inserted target antigen to generate antibodies against cellular marker p16INK4A. Sci. World J. 2012: 263737.

Li JJ, Ying RB, Yao J, Wei ZP, et al. (2011a). Risk factors and prevention of nosocomial infection after gastrointestinal cancer surgery. Chin. J. Nosocomial. 21: 4702-4704.

Li XS, Yan J, Luo B, He SJ, et al. (2011b). OY-TES-1 expression and serum immunoreactivity in glioma. China Oncology 21: 671-674.

Liang S, Wang XH, Zhao YM and Yang H (2011). Expression of cancer testis antigen related genes in human colorectal carcinomas. Chin. J. Lab. Diagnosis 15: 91-93.

Lin Q, Wang DN, Wang SX, Xie XX, et al. (2010). Expression of cancer-testis antigen MAGE-A1 in retinoblastoma and its clinical significance. Recent Adv. Ophthalmol. 30: 241-244.

Lu Y, Wei YQ, Tian L, Zhao X, et al. (2003). Immunogene therapy of tumors with vaccine based on xenogeneic epidermal growth factor receptor. J. Immunol. 170: 3162-3170.

Robbins PF, Morgan RA, Feldman SA, Yang JC, et al. (2011). Tumor regression in patients with metastatic synovial cell sarcoma and melanoma using genetically engineered lymphocytes reactive with NY-ESO-1. J. Clin. Oncol. 29: 917-924.

Scanlan MJ, Gure AO, Jungbluth AA, Old LJ, et al. (2002). Cancer/testis antigens: an expanding family of targets for cancer immunotherapy. Immunol. Rev. 188: 22-32.

Shen J, Song G, An M, Li X, et al. (2014). The use of hollow mesoporous silica nanospheres to encapsulate bortezomib and improve efficacy for non-small cell lung cancer therapy. Biomaterials 35: 316-326.

Ueda Y, Shimizu K, Itoh T, Fuji N, et al. (2007). Induction of peptide-specific immune response in patients with primary malignant melanoma of the esophagus after immunotherapy using dendritic cells pulsed with MAGE peptides. Jpn. J. Clin. Oncol. 37: 140-145.

Vonderheide RH, Hahn WC, Schultze JL and Nadier LM (1999). The telomerase catalytic subunit is a widely expressed tumor associated antigen recognized by cytotoxic T lymphocytes. Immunity 10: 673-679.

Wang CT, Zhang P, Peng F, Ruan XZ, et al. (2007). Effects of the novel tumor/testis antigen, Biot2, on proliferation of NIH3T3 cell. Xi Bao Yu Fen Zi Mian Yi Xue Za Zhi 23: 704-706.

Wang H, Zhang P and Wang CT (2009). Analysis of expression pattern of a novel testis-highly expressed gene Biot2-L and the primary study on its role in testis development. Sichuan Da Xue Xue Bao Yi Xue Ban 40: 853-856.

Xu Z, Zhang B and Chen H (2012). Progress in dendritic cell-based tumor vaccine targeting MAGE-3. Chin. J. Cancer Biother. 19: 336-340.

Yang H, Wang C, Wang R, Deng H, et al. (2009). Characterization of a novel rat gene RTAP2a, screened by cross-reactive SEREX, restrictedly expressed in testis. J. Biosci. Bioengin. 107: 589-595. 